\title{
SIMULATIONS OF THE ELECTRON CLOUD BUILDUPS AND SUPPRESSIONS IN TEVATRON AND MAIN INJECTOR*
}

\author{
Xiaolong Zhang, Jean-François Ostiguy, Weiren Chou \\ Fermilab, Batavia, IL 60510, U.S.A.
}

\begin{abstract}
To assess the effects of the electron cloud on Main Injector intensity upgrades, simulations of the cloud buildup were carried out using POSINST and compared with ECLOUD. Results indicate that even assuming an optimistic 1.3 maximum secondary electron yield, the electron cloud remains a serious concern for the planned future operational mode with 500 bunches, $3 \mathrm{e} 11$ proton per bunch. Electron cloud buildup can be mitigated in various ways. We consider a plausible scenario involving solenoids in straight section and a single clearing strip electrode (like SNEG in Tevatron) held at a potential of $500 \mathrm{~V}$. Simulations with parameters corresponding to Tevatron and Main Injector operating conditions at locations where special electron cloud detectors have been installed have been carried out and are in satisfactory agreement with preliminary measurements.
\end{abstract}

\section{INTRODUCTION}

The basic mechanism of the electron cloud effect (ECE) is well known for proton storage rings[1]. Electrons generated by beam induced ionization of the residual gas, particle loss or synchrotron radiation (e.g. LHC at CERN) on the beam pipe are accelerated across the vacuum chamber by the electrostatic field of a bunched beam. Through secondary emission resulting from electronic impact, more electrons are emitted and accelerated, eventually resulting in an avalanche effect. Saturation is reached when the beam is neutralized or when the electron space charge field near the wall surface suppresses secondary emission.

Electron cloud buildup around the beam in the vacuum chamber can reach quasi equilibrium on a relatively short time scale. The cloud can in turn interact with the beam and affect operation of the accelerator through beam loss, instability, emittance growth, vacuum pressure increase and degradation of the beam diagnostic system, etc. Deleterious effects of the electron cloud have already been observed and/or studied at proton storage rings such as PSR (LANL), RICH (BNL), SNS, SPS and LHC (CERN). Substantial resources have been invested to mitigate these problems. In principle, both the Tevatron (Tev) and Main Injector (MI) could be affected by the ECE. So far, the operational impact has been minimal due to the Main Injector's low bunch intensity and the Tevatron's large bunch spacing. Nevertheless, in view of a plan to increase the intensity of the MI much more to meet the requirements of the Proton Driver Project[2], ECE has become a major concern. Beam studies and simulations are being carried out to understand possible

*Work supported by the U.S. Department of Energy under contract No. DE-AC02-07CH11359 consequences of the ECE as the proton intensity grows in the Fermilab accelerator chain.

\section{MAIN PARAMETERS}

The main parameters relevant to the electron cloud simulations in the Tevatron and MI are summarized in Table 1. Results of the simulations will be presented and discussed the following sections.

Table 1: The main machine parameters during studies

\begin{tabular}{|l|l|l|}
\hline & \multicolumn{1}{|c|}{ Tevatron } & \multicolumn{1}{c|}{ Main Injector } \\
\hline Energy & $150 \sim 980 \mathrm{GeV}$ & $8.9 \sim 120 \mathrm{GeV}$ \\
\hline Circumference & $6283.2 \mathrm{~m}$ & $3319.4 \mathrm{~m}$ \\
\hline RF frequency & $53 \mathrm{MHz}$ & $53 \mathrm{MHz}$ \\
\hline Bunch Intensity & $4 \mathrm{e} 10 \mathrm{p}$ & $6 \mathrm{e} 10 \sim 30 \mathrm{e} 10 \mathrm{p}$ \\
\hline Filling Pattern & $30 \mathrm{bunches}$ & 504 bunches \\
\hline Bunch Spacing & $5.64 \mathrm{~m}$ & $5.64 \mathrm{~m}$ \\
\hline Bunch Length $\sigma$ & $0.51 \mathrm{~m}(150 \mathrm{GeV})$ & $0.75 \mathrm{~m}(8 \mathrm{GeV})$ \\
\hline Beam Size $\sigma$ & $1.2 \mathrm{~mm}(150 \mathrm{GeV})$ & $0.5 \mathrm{~mm}(8 \mathrm{GeV})$ \\
\hline Elliptical Beam Pipe & $12.3 \mathrm{~cm} \times 5 \mathrm{~cm}$ & $12.3 \mathrm{~cm} \times 5 \mathrm{~cm}$ \\
\hline Round Beam Pipe & $\phi-7.6 \mathrm{~cm}$ & $\phi-15.2 \mathrm{~cm}$ \\
\hline Vacuum Pressure & $20 \mathrm{nTorr}$ & $20 \mathrm{nTorr}$ \\
\hline Bend magnet $(\mathrm{T})$ & $0.6(150 \mathrm{GeV})$ & $0.1(8 \mathrm{GeV})$ \\
\hline
\end{tabular}

For the Tevatron and MI, primary electrons are generated mostly through ionization of the residual gas and beam loss. An ionization cross section of $2 \mathrm{Mbarn}$ at temperature of $300 \mathrm{~K}$ and an empirical value of about 3 lost protons /s during bunch traversal are assumed.

Since the secondary electron yield is a determinant factor, precise measurements are very important.

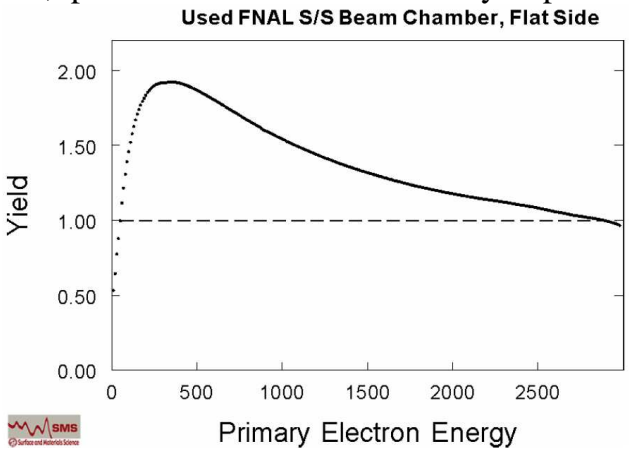

Figure 1: Secondary electron yield measured for a sample of the MI stainless steel vacuum chamber

For both Tevatron and the MI, the vacuum chamber is made out of stainless steel. Samples of beam pipe were 
sent to SLAC[3] for SEY measurement and surface composition analysis. One of the measurements is shown in Figure 1. The maximum yield $\left(\delta_{\max }\right)$ reaches 1.9 , at a corresponding incident energy of $300 \mathrm{eV}$. These values are used by the simulation code to parametrize a detailed model of the SEY behavior. To account for the fact that the SEY decreases when the surface desorbs due to baking and beam "scrubbing" via beam particles, electrons and ions, $\delta_{\max }$ is slightly scaled down and shifted toward lower energies.

\section{SIMULATIONS RESULTS}

To assess the ECE on proton driver project, extensive simulations were carried out in parallel to an experimental program. Established simulation codes such as ECLOUD, POSINST, PEI and CLOUDLAND were installed locally to support numerical investigations.

An initial assessment, including a determination of the threshold for ECE, was performed by M. Furman in 2006[4]. The additional results reported here were also obtained with POSINST.

\section{ECE in field free region}

To maximize acceptance, the MI injection region features special large aperture quadrupoles. The vacuum chamber diameter in the vicinity of these magnets is circular, with a diameter of 6 inches and a special insertion outfitted with a Retarding Field Analyzer (RAF) was installed to measure the electron flux on the chamber surface. Results of a simulation of ECE build up for a geometry corresponding to that of the test insertion are shown in Figure 2. The simulations were performed assuming a low $\delta_{\max }$ of 1.3. For a bunch intensity of $10 \mathrm{e} 10$ proton/bunch, which the MI currently achieves with slipstacked bunches, the computed electron line density grows slowly starting approximately at the $350^{\text {th }}$ bunch passage. Comparison with the electron current measured by the RFA[5] for only 84 slip-stacked bunches shows that the $\delta_{\max }=1.3$ assumed for the initial assessment was too optimistic. Setting $\delta_{\max }=1.7$ results in better agreement with measurements.



Figure 2. The started even at bunch intensity of $10 \mathrm{e} 10$ proton/bunch for low $\mathrm{SEY}=1.3$
With an intensity of $30 \mathrm{e} 10$ proton/bunch, the electron line density saturates at a level four times higher when $\delta_{\max }$ is increased from 1.3 to 1.5 .

\section{ECE in bend magnets}

Almost $95 \%$ of the MI is filled with magnets, mostly bending dipoles. Within these magnets, electrons are confined longitudinally in the vertical plane by Larmor motion. The expected consequence of this confinement is an enhancement of the ECE. Figure 3 shows the average electron density versus bunch length predicted by simulations. The full bunch length is defined as four times of the $\sigma$ of the longitudinal Gaussian bunch.

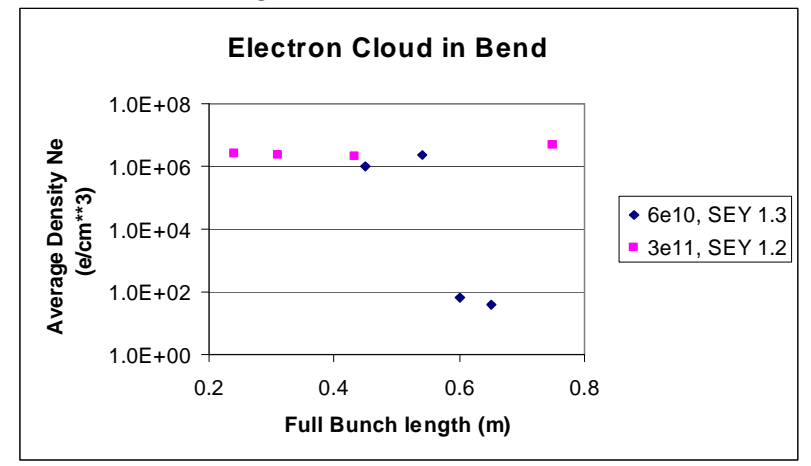

Figure 3. ECE in MI elliptical vacuum chamber within bend magnets versus the bunch length.

Even at a low proton bunch intensity of $6 \mathrm{e} 10$, the electron cloud threshold is exceeded for bunch lengths smaller than $0.54 \mathrm{~m}$. This implies that the ECE should take place during ramping, where bunch becomes shorter. This is in qualitative agreement with experimental observations. At bunch intensities such as those anticipated in the future, full neutralization is reached even for the lowest achievable $\delta_{\max }$ independently of bunch length.

\section{Effect of a clearing electrode}

A clearing electrode is a possible way to mitigate the ECE. For both the Tevatron and MI, a special SNEG electrode has been designed for test purposes. SNEG is a metal strip coated with a low activation temperature $\left(180^{\circ} \mathrm{C}\right) \mathrm{NEG}$ getter, proving distributed pumping. Kapton is used to provide electrical insulation from the vacuum pipe when a bias voltage is applied for electron clearing. This material can be used under ultra high vacuum conditions, (1e-11 Torr) and can also safely be baked at $150^{\circ} \mathrm{C}$. It can withstand approximately $80 \mathrm{KV} / \mathrm{mm}$. Overall, the design is simple, and simultaneously provides vacuum pumping and electron clearing functionalities.

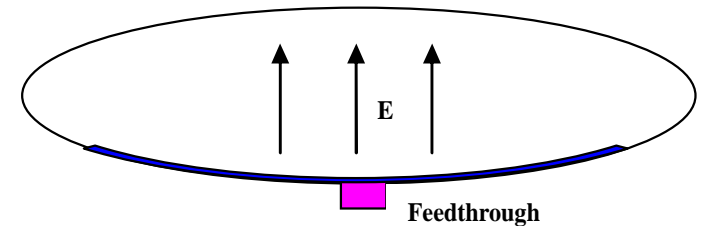

Figure 4. Clearing Electrode Design 
The electrode design is illustrated in Figure 4. The thin strip is held down by the electrical feed-throughs and by gravity. The Kapton layer providing insulation is approximately $0.5 \mathrm{~mm}$ thick. For the purposes of simulation, the electric field in the vertical plane is assumed to be uniform. While field distribution could in principle be modeled more accurately, a uniform approximation was deemed adequate, given all the other uncertainties.

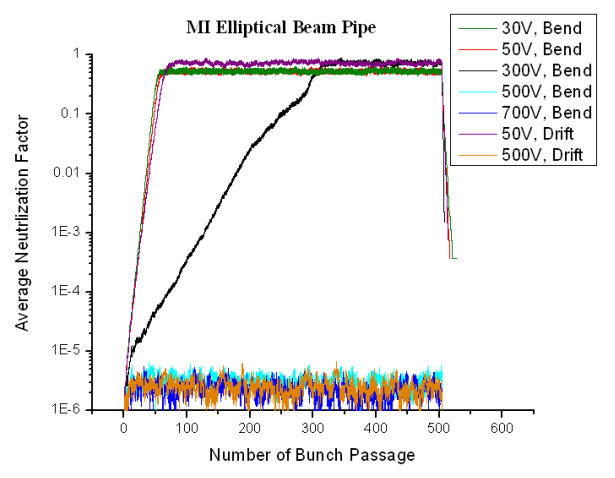

Figure 5. ECE can be suppressed by the $500 \mathrm{~V}$ clearing electrode in the beam pipe.

Calculations show that holding the clearing electrode at a potential of $500 \mathrm{~V}$ is sufficient to suppress the ECE.

\section{Effect of a solenoidal field}

The magnetic field of solenoid magnet has proved effective to suppress the ECE in field free regions at $\mathrm{KEKB}$ and in some other machines. A relatively low magnetic field confines the electrons longitudinally and prevents them from hitting the walls. Figure 6 shows the effect of a solenoidal field and compares its effectiveness to that of a clearing electrode. A field of approximately $50 \mathrm{G}$ is adequate to suppress the ECE.

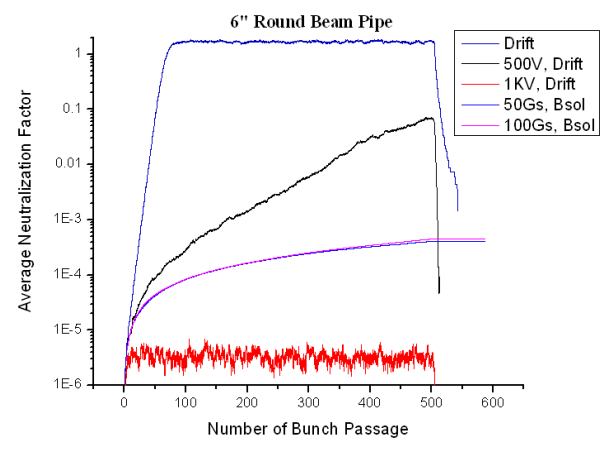

Figure 6 . The electron cloud can be suppressed by 50Gs solenoid or over $500 \mathrm{~V}$ clearing electrode

\section{Simulations for the Tevatron}

Sudden vacuum pressure rises have been observed in the Tevatron[5] in the $\mathrm{C} 0$ region, and ECE is a primary suspect. Simulation results for conditions corresponding to the ECE studies in the Tevatron are shown in Figure 7. For 30 consecutively injected bunches with observed threshold bunch intensity of $4 \mathrm{e} 10$ proton and assuming $\delta_{\max }=1.3$ everywhere, the electron density is ten times stronger in a bending magnet than a field free region. Historically, the $\mathrm{CO}$ section has experienced worse vacuum then the rest of the machine, and is plausible that due to adsorbtion, $\delta_{\max }$ is significantly higher in that region. This might explain why a significant vacuum jump was observed at $\mathrm{C} 0$ while the RFA in the specially instrumented B49 drift space detected no electron current.

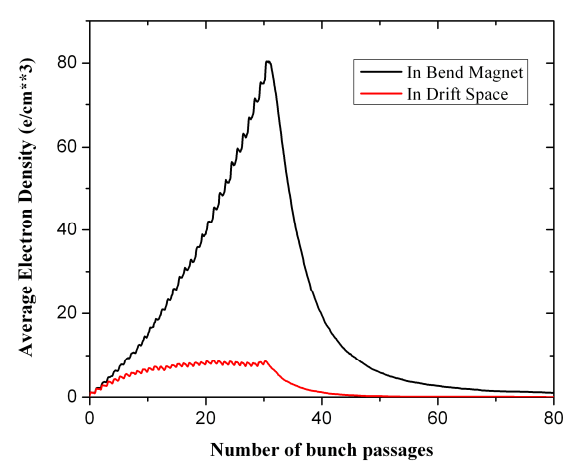

Figure 7. The ECE for 30 proton bunches in Tevatron

\section{DISCUSSIONS}

Simulations predict a bunch intensity threshold of $1 \mathrm{e} 11$ proton/bunch, assuming the lowest possible SEY (1.3) for a SS vacuum chamber. For the planned 3e11 MI intensity target, mitigation measures are essential. A combination of clearing electrodes within magnets and solenoids in field-free regions appears effective. Using NEG on the electrode material should provide some overall improvement in MI vacuum. The SNEG clearing electrodes may also prove effective in the $\mathrm{C} 0$ region of the Tevatron where the ECE and vacuum are worse.

\section{ACKNOWLEGEMENTS}

The authors are greatly indebted to Miguel Furman of LBL for support with POSINST code and his many theoretical insights. We are also grateful to Frank Zimmermann, Kazuhito Ohmi, Lanfa Wang and Kathy Harkay for valuable discussions and support.

\section{REFERENCES}

[1] M.A. Furman, "The Electron-Cloud Effect in the Arcs of the LHC", LHC Project Report 180, May 20, 1998

[2] W. Chou, "2 MW upgrade of the Fermilab Main Injector”, FERMILAB-CONF-03-145, Jun 2003

[3] Robert E. Kirby, "SEY and Surface Analysis Measurements on FNAL Main Injector Ring S/S Beam Chamber Material", SLAC-TN-06-031, Sep 18, 2006

[4] M. A. Furman, "A preliminary assessment of the electron cloud effect for the FNAL main injector upgrade", FERMILAB-PUB-05-258-AD, June 2006

[5] Xiaolong Zhang et al, "ELECTRON CLOUD STUDIES AT TEVATRON AND MAIN INJECTOR", THPAN117, this PAC07 conference, June 2007. 